\title{
Flow and sound field analysis of agricultural ultrasonic atomizing nozzle
}

\author{
Jinliang Gong ${ }^{1}$, Meixiang Wang ${ }^{1}$, Yanfei Zhang2*, Yubin Lan², Kazi Mostafa ${ }^{3}$ \\ (1. School of Mechanical Engineering, Shandong University of Technology, Zibo, 255000 China; \\ 2. School of Agricultural Engineering and Food Science, Shandong University of Technology, Zibo 255000, China; \\ 3. School of Mechanical Engineering (International Program), University of Limerick, V94 T9PX, Ireland)
}

\begin{abstract}
To solve the problem of large size of fog droplets generated in plant protection, which are not conducive to absorption by target plants and result in pollution due to excessive application, an ultrasonic atomizing nozzle suitable for agricultural plant protection was designed. First, a geometric model of the agricultural ultrasonic atomizing nozzle was established using the Design Modeler module in ANSYS FLUENT. The FLUENT simulation software program was then employed to simulate the internal flow field of the nozzle, and the internal flow field cloud image and sound pressure for various cavity depths and cavity diameters were investigated. Finally, the vapor holdup of the flow field inside the nozzle were simulated. The results indicate that the internal cavity depth and diameter of the agricultural ultrasonic atomizing nozzle affect the generation of a cavitation vortex inside the nozzle and the magnitude of the sound pressure. As the cavity depth and diameter are increased, the amplitude of sound pressure first increases and then gradually decreases. The cavity diameter has a stronger influence on the amplitude of sound pressure than the cavity depth does. The sound pressure amplitude changes marginally with the cavity depth. Simulation revealed that the ultrasonic intensity is highest and the corresponding atomization effect is strongest when the depth and diameter of the of the resonant cavity are 4 and $3 \mathrm{~mm}$, respectively. When the inlet pressure is $2 \mathrm{MPa}$, the percentage of the flow field of the ultrasonic atomizing nozzle with vapor content higher than $80 \%$ is approximately $33.94 \%$ higher than that achieved before parameter optimization. The effective space utilization rate inside the nozzle is improved.
\end{abstract}

Keywords: ultrasonic atomization, resonant cavity, sound pressure amplitude, vapor content; computational fluid dynamics DOI: $10.33440 /$ j.ijpaa.20190202.36.

Citation: Gong J L, Wang M X, Zhang Y F, Lan Y B, Mostafa K. Flow and sound field analysis of agricultural ultrasonic atomizing nozzle. Int J Precis Agric Aviat, 2019; 2(2): 32-37.

\section{Introduction}

Environmental pollution and quality problems resulting from agricultural products and caused by excessive application of pesticides are increasingly serious ${ }^{[1]}$. However, the rate of pesticide utilization is only $35 \%^{[2,3]}$. Precision application technology is an effective method of reducing the amount of pesticide residues, and agricultural nozzles are the crucial components of such technology ${ }^{[4-6]}$. Various types of agricultural nozzles are available, and the large size of fog droplets generated by pressure nozzles is not conducive to absorption by target plants. The fog droplets generated using centrifugal atomization nozzles are small; however, these nozzles have some disadvantages, such as a complex structure, high cost, and spraying contour with a hollow cone angle ${ }^{[7-11]}$. Droplet size is a key factor affecting spray quality and the plant protective effect. As the size of fog droplets is decreased, the number of fog droplet increases and the probability of the pesticide hitting its target will also increases. The coverage is more uniform and the fog droplets have higher adhesion ability when they are

\section{Received date: 2019-11-18 Accepted date: 2019-12-15}

Biographies: Jinliang Gong, $\mathrm{PhD}$, associate professor, research interests: intelligent agricultural robot technology and equipment, Email: gjlwing@qq.com; Meixiang Wang, Postgraduate student, research interests: precision agricultural technology, Email: 1604688804@qq.com; Yubin Lan, PhD, Distinguished Professor, Director, research interests: precision agricultural aviation application, Email: ylan@scau.edu.cn; Kazi Mostafa, PhD, research interests: robotics, image processing, Email: kazi.mostafa@live.com.

*Corresponding author: Yanfei Zhang, $\mathrm{PhD}$, professor, research interests: intelligent agricultural robot technology and equipment. School of agricultural engineering and food science, Shandong university of Technology, Zibo 255000, China. Email: 1392076@sina.com. smaller $^{[12-15]}$. For contact-killing pesticides that are nonconductive in plant leaf tissue, a small droplet size can considerably improve the control effect ${ }^{[16]}$.

An ultrasonic atomizing nozzle has a simple structure, can spray uniform particles several micrometers in diameter, and is widely used in numerous fields such as dust reduction and humidification $^{[17-19]}$. Li et al. ${ }^{[20]}$ (2017) analyzed the influence of different structural parameters on the spray velocity of ultrasonically excited nozzles; Zhang et al. ${ }^{[21]}$ (2017) analyzed and researched the internal flow field of ultrasonic atomizing nozzles at different spraying speeds; $\mathrm{Li}^{[22]}$ (2014) studied the different types of hydrodynamic ultrasonic atomizing nozzles Atomized droplet size under structural parameters; Liu et al. ${ }^{[23]}$ (2018) studied the internal flow field cloud diagram and gas phase distribution of the ultrasonic atomizing nozzle under different gas content in; $\mathrm{Li}$ et al. ${ }^{[24]}$ (2019) analyzed the droplet size of gas-liquid two-phase nozzle under different spraying pressure in.

No ultrasonic atomizing nozzle has yet been designed specifically for pesticide spraying by plant protection drones. On the basis of computational fluid dynamics, the working principles of ultrasonic atomizing nozzles, and the atomization requirement of pesticide spraying, this study used the FLUENT model in ANSYS (ANSYS is headquartered in Pittsburgh, Pennsylvania, USA) to design an ultrasonic atomizing nozzle model for agricultural plant protection by using an UAV. The flow field was simulated and analyzed to investigate the flow field distribution of the resonant cavity of the ultrasonic atomizing nozzle under different structural parameters. In this manner, such field analysis was used to determine the variation in sound pressure amplitude for varying structural parameters of the 
resonant cavity. The findings provide a theoretical basis for application of ultrasonic atomizing nozzles on UAVs used for the agricultural plant protection.

\section{Materials and methods}

\subsection{Working principle of ultrasonic atomization}

Ultrasonic atomization is a process of cavitation in which ultrasonic energy is used to create small droplets from a liquid. Cavitation is the dynamic process of growth and collapse of micro gas core cavitation bubbles in a liquid once the sound pressure has reached certain value ${ }^{[20-21]}$. When ultrasonic waves act on the interior of the liquid, a partial negative pressure region is generated. When the negative pressure overcomes the bonding force between the liquid molecules, the liquid is segmented to form cavitation bubbles. These bubbles collapse under the vibration of the ultrasonic waves, and a strong shock wave is generated around the bubbles to atomize the liquid ${ }^{[22,23]}$.

\subsection{Building and meshing of a geometric model}

The geometric model of the agricultural ultrasonic atomizing nozzle (Figure 1) was established using the Design Modeler module in the fluid mechanics software FLUENT. The liquid flows in from the inlet pipe, is accelerated along the inclined wall of the nozzle, and is then ejected from the high-speed jet hole into the resonance cavity. The ultrasonic wave generated has a cavitation effect on the liquid, causing the liquid to be segmented and atomized into uniform small droplets, which are then ejected through the outlet. The geometric model parameters are as follows: the diameter of the orifice $(d)$ is $1.5 \mathrm{~mm}$, diameter of the liquid inlet pipe is $10 \mathrm{~mm}$, distance between the high-speed orifice and resonant cavity is $3 \mathrm{~mm}$, and diameter of the liquid outlet is $2 \mathrm{~mm}$. The influence of two key parameters, the cavity diameter $D$ and cavity depth $l$, on the size of droplets produced by the ultrasonic atomizer was investigated.

The calculation domain of the geometric model of the agricultural ultrasonic atomizing was meshed using Mesh. The number of grid squares was 4524 , and the number of nodes was 2406. The meshing result is illustrated in Figure 2.

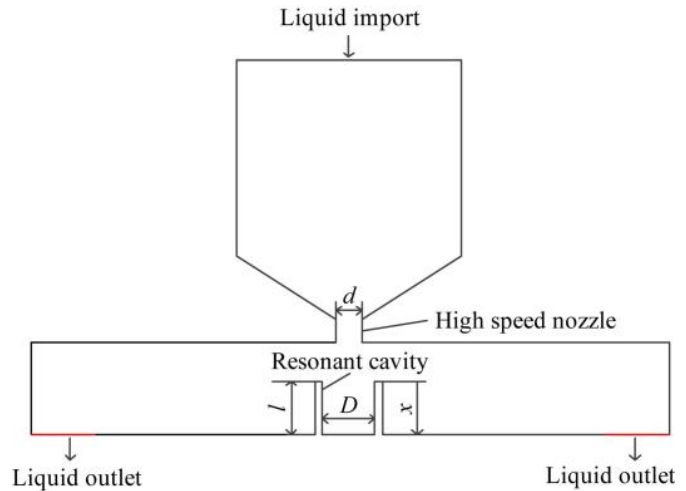

Figure 1 Schematic diagram of the agricultural ultrasonic atomizing nozzle

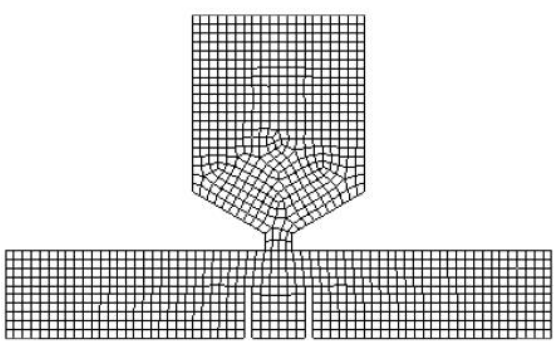

Figure 2 Grid map

\subsection{Flow field simulation}

According to the structural characteristics of the agricultural ultrasonic atomizer, the liquid to be sprayed was assumed to be ideal and incompressible. The control equation was discretized using the finite volume method, and the flow field was calculated using the simple unstructured grid algorithm. In the FLUENT module of ANSYS 15.0, the solver model employed was polyphase Euler and the viscous standard $k-\varepsilon$ model. The basic control equations of the model are the mass, momentum, and energy conservation equations. The kinetic energy equation and diffusion equation of the turbulence model meet the following requirements:

$$
\begin{gathered}
\frac{\partial(\rho k)}{\partial t}+\frac{\partial\left(\rho k u_{i}\right)}{\partial x_{i}}=\frac{\partial}{\partial x_{j}}\left[\left(\mu+\frac{\mu_{t}}{\sigma_{k}}\right) \frac{\partial k}{\partial x_{j}}\right]+G_{k}+G_{b}-\rho-Y_{M}+S_{K} \\
\frac{\partial(\rho \varepsilon)}{\partial t}+\frac{\partial\left(\rho \varepsilon \mu_{i}\right)}{\partial x_{i}}=\frac{\partial}{\partial x_{j}}\left[\left(\mu+\frac{\mu_{t}}{\sigma_{\varepsilon}}\right) \frac{\partial \varepsilon}{\partial x_{j}}\right]+C_{1 \varepsilon} \frac{\varepsilon}{k}\left(G_{k}+C_{\varepsilon} G_{b}\right)- \\
C_{2 \varepsilon} \rho \frac{\varepsilon}{k}+S_{\varepsilon}
\end{gathered}
$$

where, $\rho$ is the fluid density; $k$ is the turbulent flow energy; $\varepsilon$ is the turbulent flow energy dissipation rate; $\mu$ is the dynamic viscosity of the fluid; $G_{K}$ is the turbulent flow energy produced by the laminar velocity gradient; $G_{b}$ is the turbulent flow energy generated by buoyancy, and $Y_{M}$ is the coefficient of influence of turbulent pulsation expansion on the total dissipation rate. The model constants are as follows: $\sigma_{k}=1.0, C_{1 \varepsilon}=1.44, C_{2 \varepsilon}=1.92$, and $\sigma_{\varepsilon}=1.3$.

The turbulence speed $\mu_{t}$ is given as follows:

$$
\mu_{t}=\rho C_{\mu} \frac{k^{2}}{\varepsilon}
$$

Where the model constant $C_{\mu}=0.09$.

\subsection{Sound field simulation}

The Fowcs Williams and Hawkings (FW-H) model was employed for acoustic simulation. The FW-H equation is given as follows:

$$
\begin{aligned}
& \left(\frac{\partial^{2}}{c^{2} \partial t^{2}}-\frac{\partial^{2}}{\partial x_{i}^{2}}\right) p\left(x_{i}, t\right)=\frac{\partial^{2}}{\partial x_{i} \partial x_{j}}\left[T_{i j} H(f)\right]+ \\
& \frac{\partial}{\partial t}\left\{\left[\rho_{0} v_{n}+\rho\left(\mu_{n}-v_{n}\right)\right] \delta(f)\right\}- \\
& \frac{\partial}{\partial x_{i}}\left\{\left[-P_{i j} n_{j}+\rho u_{i}\left(\mu_{n}-v_{n}\right)\right] \delta(f)\right\}
\end{aligned}
$$

where, $p\left(x_{i}, t\right)$ is the sound pressure intensity of the observation point at time $t ; \rho, \mu_{i}$ and $P_{i j}$ are the density, velocity, and stress tensor, respectively; $v_{n}$ is the surface velocity component perpendicular to the surface of the sound source surface; $T_{i j}$ is the Lighthill tensor; $\delta_{i j}$ is the Roneck symbol; $H(f)$ is the Heaviside function; $\delta(f)$ is the Dirac function; and $c$ is the speed of sound in $\mathrm{m} / \mathrm{s}$.

\section{Results and discussion}

\subsection{Effect of cavity depth on the nozzle flow field}

On the basis of the polyphase Euler and viscous standard $k-\varepsilon$ model parameters, the internal flow field of agricultural ultrasonic atomizing nozzles with different cavity depths was simulated. The inlet pressure was selected to be $2 \mathrm{MPa}$; the cavity diameter $(D)$ was selected to be $4 \mathrm{~mm}$, and analyze the velocity streamline diagram was obtained inside and near the cavity when the cavity depth was $l=2,3,4$, and $5 \mathrm{~mm}$ (Figure 3). Once the liquid is ejected from the high-speed nozzle, it quickly enters the cavity. The liquid continuously rushes along both sides of the cavity and collides with the injected liquid to generate a shock wave. An 
increase in sound pressure causes the formation of a cavitation vortex inside the flow field, and the cavitation bubbles collapse under the action of the ultrasonic wave, enhancing the atomization effect of the liquid.

The velocity streamline diagrams obtained using different cavity depths reveal that when the cavity depth was $l=2 \mathrm{~mm}$, fluid was injected into and rushed out of the cavity; however, no cavitation vortex formed. When the cavity depth was $l=5 \mathrm{~mm}$, the cavity was too deep, and forcing the fluid into the bottom of the cavity was difficult. A cavitation vortex formed far away from the bottom of the cavity, and the space utilization rate was low. When the depth of the cavity was $l=3$ and $4 \mathrm{~mm}$, more fluid moved in and out of the cavity and a cavitation vortex formed near the bottom of the cavity. Therefore, the space utilization rate was high. A cavity depth of $l=4 \mathrm{~mm}$ resulted in a spatiotemporal vortex with the best shape and the optimal cavitation effect.

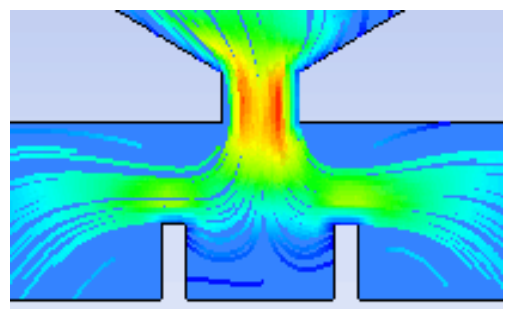

a. $l=2 \mathrm{~mm}$

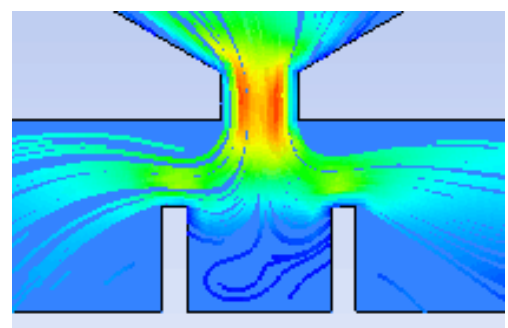

b. $l=3 \mathrm{~mm}$

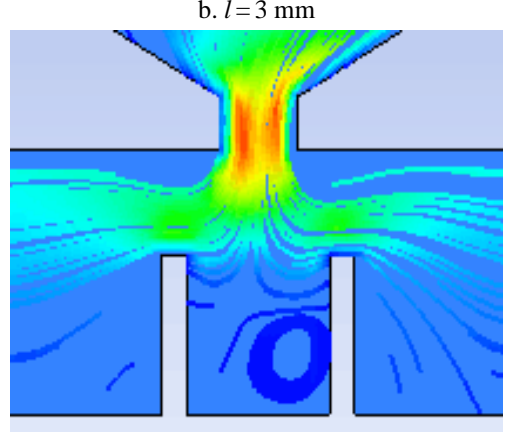

c. $l=4 \mathrm{~mm}$

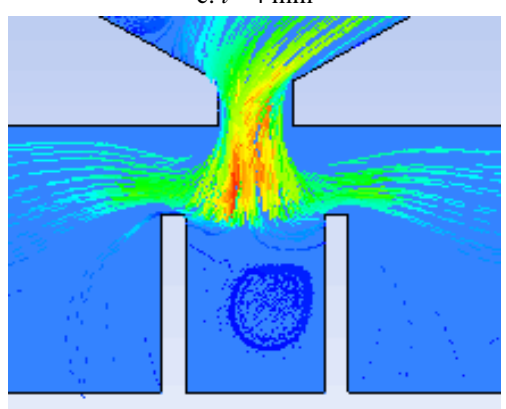

d. $l=5 \mathrm{~mm}$

Figure 3 Velocity streamline diagrams for different cavity depths

The upper plane of the cavity was defined as the interface with a depth of 0 . The trend in the internal pressure of the cavity for a cavity depth $x$ is displayed in Figure 4. With an increase in the cavity depth $x$, the pressure increased gradually. The comparison curve indicates that when $l=2 \mathrm{~mm}$, the rate of change of the pressure with the cavity depth was low, the ultimate pressure at the bottom of the cavity was $1.21 \mathrm{MPa}$, and the pressure difference was $0.17 \mathrm{MPa}$. Because the depth of the cavity was too small, the liquid flowed out quickly once it had completely filled the bottom of the cavity, and the difference in pressure between the cavity opening and bottom was too small. Thus, cavitation bubble was not generated. Consequently, the atomization effect was weak. When $l=5 \mathrm{~mm}$, the pressure difference between the cavity opening and bottom was too large, which made it difficult to force the fluid into the bottom of the cavity. When $l=3,4 \mathrm{~mm}$, the trends in pressure with cavity depth were essentially the same. The pressure became stable after increasing rapidly, and the limit pressure at the bottom of the cavity and initial pressure difference in the cavity were 0.31 and $0.32 \mathrm{MPa}$ respectively. The liquid was injected into the cavity and an ultrasonic wave was generated, which enhanced the liquid atomization effect.

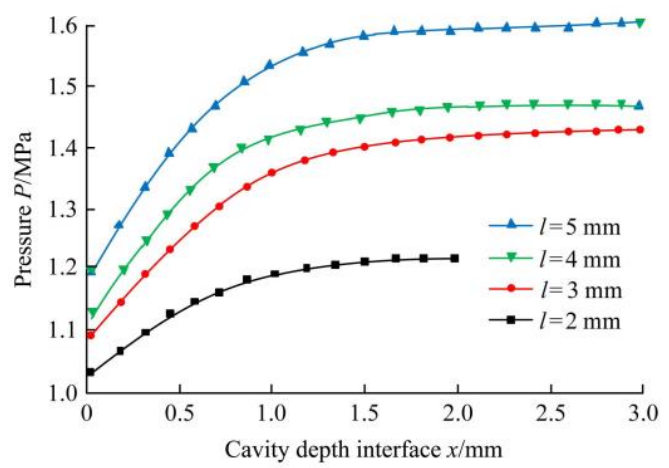

Figure 4 Curve of pressure changing with cavity depth interface under different cavity depth

\subsection{Effect of cavity depth on the nozzle sound field}

The FW-H model was selected for acoustic simulation. Figure 5 displays the amplitude curve of the sound pressure when the inlet pressure $(P)$ was $2 \mathrm{MPa}$, diameter of the cavity $(d)$ was $4 \mathrm{~mm}$, and depth $l$ of the cavity was 2, 3, 4 and $5 \mathrm{~mm}$. Under constant inlet pressure, different cavity depths correspond to different sound pressure amplitudes. When $l$ was between 2 and $4 \mathrm{~mm}$, the sound pressure amplitude increased with an increase in the cavity depth. When $l$ was between 3 and $4 \mathrm{~mm}$, the sound pressure amplitude increased marginally. When $l$ was between 4 and $5 \mathrm{~mm}$, the sound pressure amplitude decreased with an increase in cavity depth. The higher the sound pressure, the higher was the ultrasonic intensity and the stronger was the atomization effect. Therefore, the optimal atomization effect was achieved when the cavity depth was $l=4 \mathrm{~mm}$. The amplitude of the sound pressure reached $0.013 \mathrm{MPa}$.

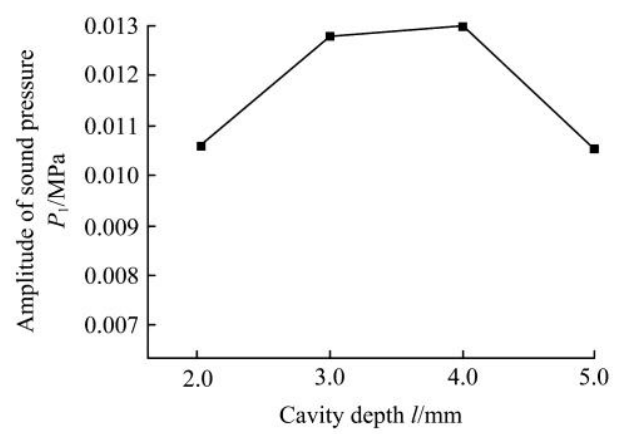

Figure 5 Sound pressure amplitude versus cavity depth

\subsection{Influence of cavity diameter on the flow field and sound field}

Figure 6 displays the velocity flow inside and around the 
cavity for an inlet pressure of $2 \mathrm{MPa}$, cavity depth $(l)$ of $4 \mathrm{~mm}$, and cavity diameters $(D)$ of $2,3,4$, and $5 \mathrm{~mm}$. When the cavity diameter was $2 \mathrm{~mm}$, most of the liquid flowed out from the periphery of the resonant cavity; only a small part of the liquid entered and then left the resonant cavity. When the diameter of the cavity was 3 and $4 \mathrm{~mm}$, the liquid poured into and then left the cavity. The liquid molecules collided to generate ultrasonic waves and consequently form a cavitation vortex. When $D=3 \mathrm{~mm}$, the strongest cavitation vortex effect was achieved, and a complete cavitation vortex was formed on both the left and right sides of the cavity. When the diameter of the cavity was $D=5 \mathrm{~mm}$, because the cavity diameter was too large, most of the liquid was directly injected into the cavity and then rushed out along the walls of the cavity without undergoing collisions; thus, no vortex was generated.

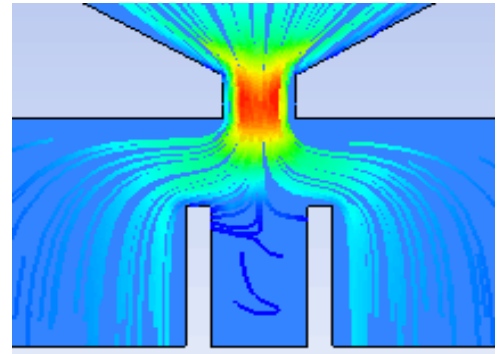

a. $D=2 \mathrm{~mm}$

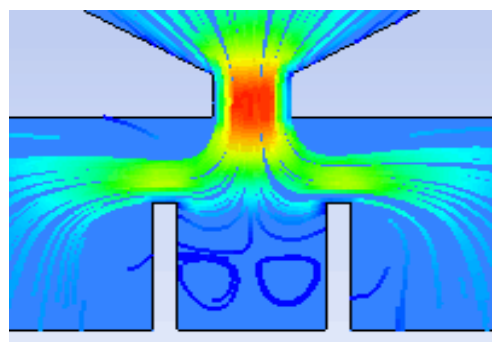

b. $D=3 \mathrm{~mm}$

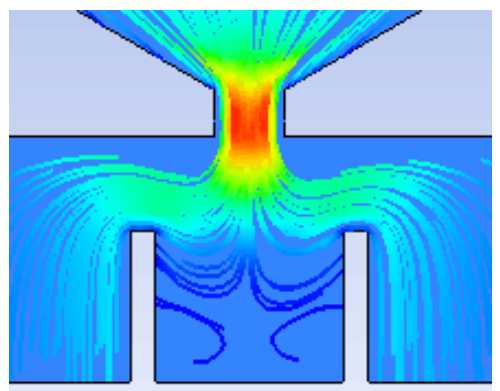

c. $D=4 \mathrm{~mm}$

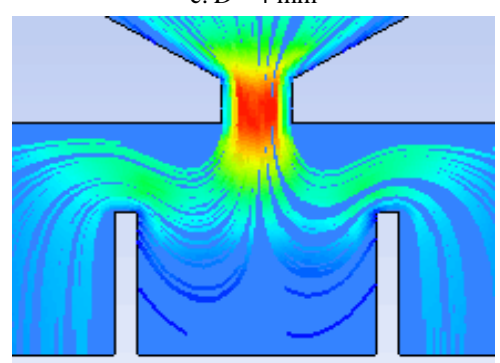

d. $D=5 \mathrm{~mm}$

Figure 6 Velocity streamline diagrams for different resonator diameters

Figure 7 depicts the variation in the pressure with the cavity depth when the cavity diameter was $2,3,4$, and $5 \mathrm{~mm}$. When $D=$ $2 \mathrm{~mm}$, the pressure increased slowly as the cavity depth was increased from 0 to $1.4 \mathrm{~mm}$. When $1.4 \mathrm{~mm}<x<4 \mathrm{~mm}$, the pressure was relatively constant. The pressure at the bottom of the cavity reached $1.95 \mathrm{MPa}$ because the cavity diameter was too small and filling the cavity with the fluid was difficult. Therefore, the intensity of the generated ultrasonic waves was low. When $D=3$ and $4 \mathrm{~mm}$, the pressure changed with the cavity depth similar to that for $D=2 \mathrm{~mm}$; however, the final pressure at the bottom of the cavity was lower when $D=3$ and $4 \mathrm{~mm}(1.79$ and $1.68 \mathrm{MPa})$ than when $D=2 \mathrm{~mm}(1.95 \mathrm{MPa})$. The liquid was smoothly injected into the resonant cavity, and the liquid flowing in collided with that flowing out, generating shock waves and promoting the formation of a cavitation vortex. When the diameter of the resonant cavity was $5 \mathrm{~mm}$, the pressure curve changed substantially. The pressure difference between the bottom and top of the resonant cavity was $0.65 \mathrm{MPa}$, and the final pressure at the bottom of the resonant cavity was1.61 $\mathrm{MPa}$. At this time, the liquid was directly poured into the resonant cavity and flowed out along the cavity's walls. The liquid flowing into and out of the cavity did not collide. Therefore, no shock waves were generated and the atomization effect was weak.

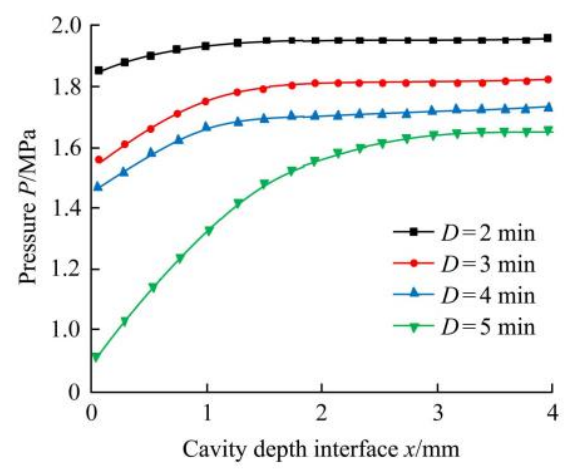

Figure 7 Pressure versus cavity depth for different cavity diameters

Figure 8 illustrates the amplitude curve of the acoustic pressure of the agricultural ultrasonic atomizing nozzle when the inlet pressure was $2 \mathrm{MPa}$; Cavity depth was $4 \mathrm{~mm}$; and cavity diameter was $2,3,4$, and $5 \mathrm{~mm}$. In the case of constant inlet pressure, different cavity diameters correspond to different sound pressure amplitudes. When the cavity diameter was less than $3 \mathrm{~mm}$, the amplitude of the sound pressure increased with an increase in the cavity diameter. When the cavity diameter $D$ was greater than $3 \mathrm{~mm}$, the amplitude of the sound pressure decreased with an increase in the cavity diameter. When $D=2 \mathrm{~mm}$, less liquid poured into the resonance cavity, and most of the liquid flowed out from the surrounding of the resonance cavity, which resulted in weak ultrasonic intensity and low-amplitude sound pressure. When the diameter of the cavity was $3 \mathrm{~mm}$, a considerable amount of liquid was injected into and rushed out of the cavity and the incoming and outgoing liquid collided to generate shock waves. The ultrasonic intensity was high, and the sound pressure amplitude was large. When $D=5 \mathrm{~mm}$, because the cavity diameter was too large, most of the liquid was directly injected into the cavity and then flowed out along the walls of the cavity without undergoing collision, which resulted in weak ultrasonic intensity and low-amplitude sound pressure.

\subsection{Simulation analysis of the nozzle atomization effect}

According to the above simulation analysis of the flow field and sound field inside the nozzle, when the diameter of the nozzle is $1.5 \mathrm{~mm}$, the optimal structural parameters of the resonant cavity can be concluded to be as follows: depth of the resonant cavity $l=4 \mathrm{~mm}$ and the diameter of the resonant cavity $D=3 \mathrm{~mm}$. For an inlet pressure $(P)$ of $2 \mathrm{MPa}$, cavitation gas phase simulation was performed on the flow field inside the ultrasonic atomizing nozzle 
before and after structural parameter optimization; the results are illustrated in Figure 9. The volume of the cavity that held more than $80 \%$ vapor was considerably increased compared with the volume when non optimized parameters were used. The cavitation bubbles near the nozzle were small, and as the bubbles in the fluid moved in a nonlinear vibration mode, they began to gradually accumulate and form a larger bubble volume. When the bubbles burst, the sound energy was converted into jet energy and local pressure energy to strengthen the atomization effect of the nozzle.

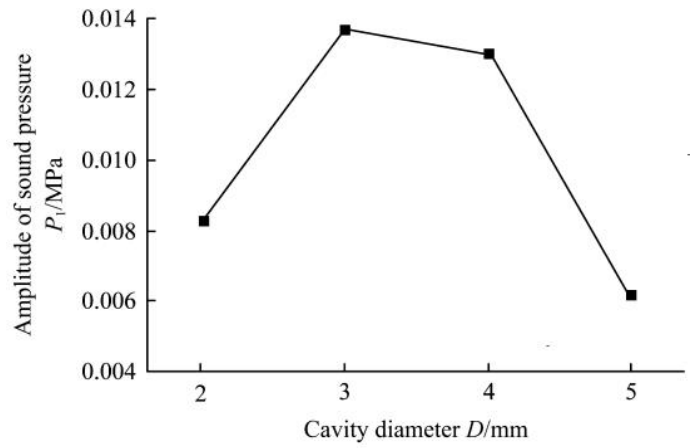

Figure 8 Sound pressure amplitude versus cavity diameter

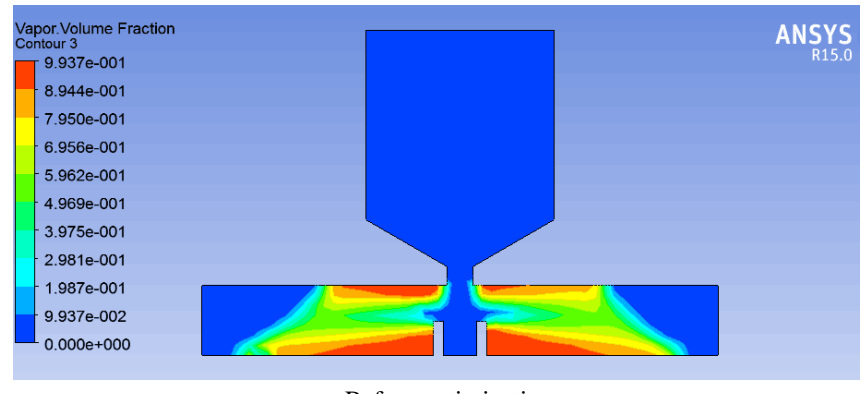

a. Before optimization

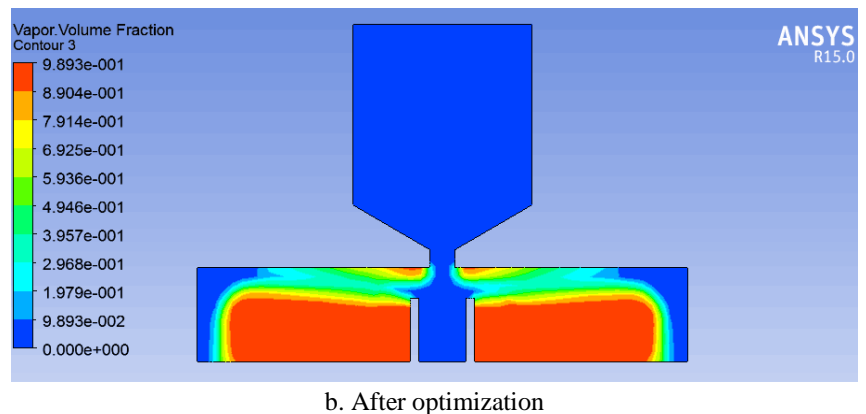

Figure 9 Cloud map of the steam rate

Figure 10 presents a comparison of the percentage of volume with steam content higher than $80 \%$ before and after optimization of the structural parameters. When the inlet pressure was $2 \mathrm{MPa}$, this percentage before the optimization of the structural parameters was only $45.27 \%$ of the flow field below the nozzle hole. After the optimization of the structural parameters, the percentage was $79.21 \%$ of the volume under the nozzle hole, which represents an increase of $33.94 \%$. When the inlet pressure was $3 \mathrm{MPa}$, the percentage of volume with steam content higher than $80 \%$ before and after optimization was $51.41 \%$ and $82.76 \%$, respectively, which represents an increase of $31.35 \%$ after optimization.

In order to simulate the atomization process, this paper uses the fluid calculation software Fluent to carry out the numerical analysis of the secondary atomization. Figure 11 is a cloud distribution diagram of the mist droplets when the inlet pressure is $2 \mathrm{MPa}$. The droplet deposition area is located directly below the nozzle. The ultrasonic atomizing nozzle has no spray profile with a hollow cone angle, so the spraying effect of the ultrasonic atomizing nozzle is better than that of the centrifugal nozzle. Figure 12 is a volume distribution diagram of atomized particles with particle size. It can be seen from the figure that the droplet particles are usually concentrated in the range of $25 \mu \mathrm{m}$ to $70 \mu \mathrm{m}$. Within this range, the volume percentage of the droplets increases first, and then decreases as the particle size increases. When the inlet pressure is $2 \mathrm{MPa}$, the volume occupied by the droplet diameter of $50 \mu \mathrm{m}$ is the largest. When the inlet pressure is $3 \mathrm{MPa}$, the volume occupied by the droplet diameter of $40 \mu \mathrm{m}$ is the largest. Therefore, the atomizing particle diameter of the ultrasonic atomizing nozzle is smaller than that of the pressure nozzle (80$200 \mu \mathrm{m})$.

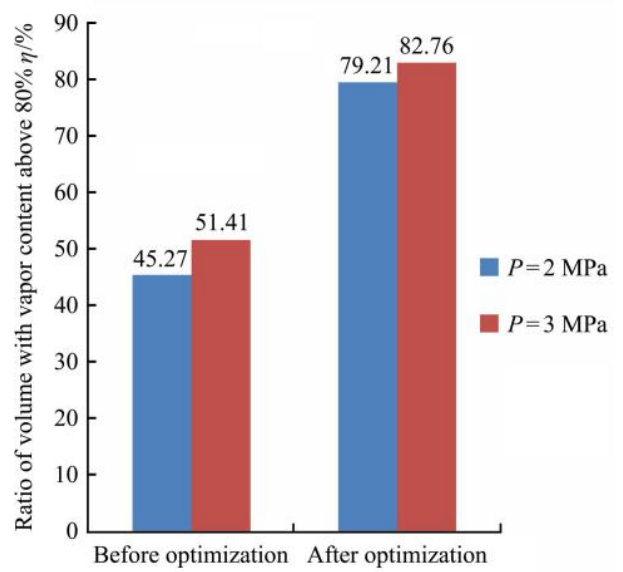

Figure 10 Percentage of volume with steam content higher than $80 \%$ before and after optimization

particle-tracks-1

Particle diameter/m

$8.66 \mathrm{e}-05$

$8.34 \mathrm{e}-05$
$8.02 \mathrm{e}-05$

$8.00 \mathrm{e}-05$

$7.38 \mathrm{e}-05$

$7.06 \mathrm{e}-05$
$7.04 \mathrm{e}-05$
$6.42 \mathrm{e}-05$

$6.42 \mathrm{e}-05$
$6.10 \mathrm{e}-05$
$6.08 \mathrm{e}-05$

$6.10 \mathrm{e}-05$

$6.08 \mathrm{e}-05$
$5.46 \mathrm{e}-05$

$5.46 \mathrm{e}-05$
$5.14 \mathrm{e}-05$

$5.02 \mathrm{e}-05$
5

$4.50 \mathrm{e}-05$

4. $18 \mathrm{e}-05$

$4.06 \mathrm{e}-05$

$3.55 \mathrm{e}-05$

$3.23 \mathrm{e}-05$
$3.01 \mathrm{e}-05$
3.5

$3.01 \mathrm{e}-05$
$2.59 \mathrm{e}-05$

$2.59 \mathrm{e}-05$
$2.27 \mathrm{e}-05$

Figure 11 Cloud distribution of mist droplets

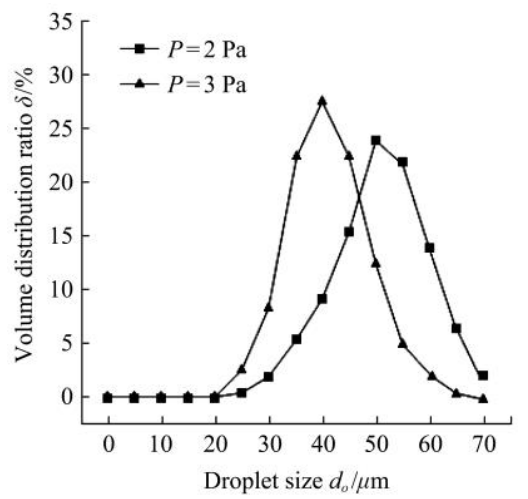

Figure 12 Volume distribution of atomized particles with particle size

\section{Conclusion}

(1) Using flow field simulation analysis, the velocity flow 
diagrams, pressure curves, and sound pressure amplitude curves of different local resonators were compared. When the inlet pressure was constant, the diameter of the nozzle was $1.5 \mathrm{~mm}$, the depth of the resonator $(l)$ was $4 \mathrm{~mm}$, and the diameter of the resonator $(D)$ was $3 \mathrm{~mm}$, a clear cavitation vortex was formed in the resonator and the largest sound pressure amplitude and optimal atomization effect were achieved.

(2) Simulation analysis of the vapor holdup of the flow field inside the nozzle for an inlet pressure of $2 \mathrm{MPa}$ indicated that the percentage of the distribution volume with the vapor content higher than $80 \%$ in the flow field of the ultrasonic atomizing nozzle was $33.94 \%$ higher after the optimization of the structural parameters. The effective space utilization ratio inside the nozzle also increased after optimization. The cavitation bubbles near the nozzle were small, and as the fluid flow bubbles moved in a nonlinear vibration mode, they gradually accumulated and collapsed. By converting the sound energy into jet energy and local pressure energy, the atomization effect of the nozzle was strengthened.

\section{Acknowledgments}

This work was funded by the National Natural Science Foundation of China (61303006), Top Talents Program for One Case One Discussion of Shandong Province, National Key Research and Development Program in Shandong Province (2019GNC106127).

\section{[References]}

[1] Lan Y B, Peng J, Jin J. Research status and development of pesticide spray particle size. Journal of South China Agricultural University, 2016, 37(06): 1-9. doi: 10.7671/j.issn.1001-411X.2016.06.001. (in Chinese)

[2] Yuan H Z, Guo Y W, Xue X Y, Yan X J, Chen C, Kong X, et al. The promotion and application of plant protection unmanned aircraft to improve the utilization rate of pesticides in China. Agricultural Engineering Technology, 2018, 38(09): 46-50. doi: 10.16815/ j.cnki.11-5436/s.2018.09.008. (in Chinese)

[3] Zhang F G, Hong T S, Wang J J, Chen J Y, Lv J T. Research progress in modern pesticide spraying technology and equipment. Agricultural Mechanization Research, 2011, 33(02): 209-213. doi: 10.13427/ j.cnki.njyi.2011.02.035. (in Chinese)

[4] Song L J, Li J P, Yang X, Wang P F, Liu H J. Research progress in pesticide application technology of plant protection drones. Modern Agricultural Science and Technology, 2019, (11): 125-128. doi: 10.3969/j.issn.1007-5739.2019.11.077. (in Chinese)

[5] He Y, Xiao S F, Fang H, Dong T, Tang Y, Nie P C, et al. Development status and application decision of plant protection UAV application nozzle. Transactions of the Chinese Society of Agricultural Engineering, 2018, 34(13): 113-124. doi: 10.11975/j.issn.1002-6819.2018.13.014. (in Chinese)

[6] Fan R, Shi S B, Yang F Z, Zhao Y L, Liu Z J, Huang F G. Research Status and Development Trend of Common Sprinklers for Plant Protection Machinery in China. Journal of Agricultural Mechanization Research, 2014, 36(06): 6-9. doi: 10.13427/j.cnki.njyi.2014.06.002 . (in Chinese)

[7] Dong F L, Zhou H P. Progress in the development of foreign plant protection nozzle technology. Journal of Jiangxi Agricultural University, 2018, 40(04): 866-874. doi: 10.13836/j.jjau.2018109. (in Chinese)

[8] Ru Y, Zhu C Y, Bao R, Li Z F, Ding T. Particle size distribution of airborne plant protection nozzles in wind tunnel and flight conditions. Transactions of the Chinese Society of Agricultural Engineering, 2016, 32(20): 94-98. doi: 10.11975/j.issn.1002-6819.2016.20.012. (in Chinese)

[9] Wen S, Lan Y B, Zhang J T, Li S H, Zhang H Y, Xing H. Analysis and test of atomization characteristics of ultra-low capacity swirl nozzle of agricultural UAV. Chinese Journal of Agricultural Engineering, 2016,
32(20): 85-93. doi: 10.11975/j.issn.1002-6819.2016.20.011. (in Chinese)

[10] Liu F L, Zhang X H, Ma W W, Liu X M. Development status of large-scale plant protection machinery and application technology in foreign countries. Journal of Agricultural Mechanization Research, 2010, 32(03): 246-248+252. doi: 10.13427/j.cnki.njyi.2010.03.033. (in Chinese)

[11] Zhou Q Q, Xue X Y, Qian S Y, Qin W C. The current status and research direction of aviation nozzles. Chinese Journal of Agricultural Mechanization, 2016, 37(10): 234-237. doi: 10.13733/ j.jcam.issn.2095-5553.2016.10.049. (in Chinese)

[12] Zhang H C, Zheng J Q, Zhou H P, DORR G J. Study on droplet deposition and off-target drift during pesticide spraying. Transactions of the Chinese Society of Agricultural Machinery, 2017, 48(08): 114-122. doi: 10.6041/j.issn.1000-1298.2017.08.012. (in Chinese)

[13] Xu D J, Xu G C, Xu X L, Gu Z Y. Effects of liquid application rate, droplet size, blade inclination and additives on the deposition of pesticides on rice leaves. Southwest China Journal of Agricultural Sciences, 2015 , 28(05): 2056-2062. doi: 10.16213/j.cnki.scjas.2015.05.038. (in Chinese)

[14] Wu X Q, Zhao X Y, Xu Y Z, Wang J N, Zhou F Y, Zhou H Z, et al. Research progress in precise application techniques for plant biological control. China Agricultural Science and Technology Review, 2019, 21(03): 13-21. doi: 10.13304/j.nykjdb.2018.0292. (in Chinese)

[15] Li J Y, Lan Y B, Shi Y Y. The characteristics of airflow of rotorcraft and the research progress of field application. Transactions of the Chinese Society of Agricultural Engineering, 2018, 34(12): 104-118. doi 10.11975/j.issn.1002-6819.2018.12.013. (in Chinese)

[16] Yuan H Z, Wang G B. Relationship between droplet size and coverage density and pesticide control effects. Plant Protection, 2015, 41(06): 9-16. doi: 10.3969/j.issn.0529-1542.2015.06.002 . (in Chinese)

[17] Yang X Y, Xiao F. Design of ultrasonic atomizing nozzle. Automobile Practical Technology, 2019(05): 152-153. doi: 10.16638/ j.cnki.1671-7988.2019.05.047. (in Chinese)

[18] Mo R Y, Lin S Y, Wang C H. Research methods and progress of ultrasonic cavitation. Applied Acoustics, 2009, 28(05): 389-400. doi: 10.11684/j.issn.1000-310X.2009.05.012. (in Chinese)

[19] Cheng X R, Zhang S Y, Fang N. Advances in the application of ultrasonic cavitation technology in the chemical industry. Applied Chemicals, 2018, 47(08): 1753-1757. doi: 10.16581/ j.cnki.issn1671-3206.2018.08.029. (in Chinese)

[20] Li H X, Liu Q Z, Liu Y P, Zhang J L. Numerical simulation of atomization characteristics of ultrasonic excited nozzle based on CFD. Journal of Vacuum Science and Technology, 2017, 37(01): 113-117. doi: 10.13922/j.cnki.cjovst.2017.01.20 (in Chinese)

[21] Zhang J, Peng Y T, Liu Q Z. Simulation of Flow Field Characteristics of Ultrasonic Atomizing Nozzle Based on CFD. China Powder Science and Technology, 2017, 23(02): 20-23. doi: 10.13732/ j.issn.1008-5548.2017.02.004 . (in Chinese)

[22] Li L. Research on adaptability of hydrodynamic ultrasonic atomizing drainage gas recovery technology. Western Exploration Engineering, 2014, 26(12): 11-13. doi: 10.3969/j.issn.1004-5716.2014.12.004. (in Chinese)

[23] Liu Q Z, Li H X, Liu Y P. Numerical simulation of flow field in gas-liquid two-axis ultrasonic nozzle. Machine Tool \& Hydraulics, 2018, 46(19): 122-124. doi: 10.3969/j.issn.1001-3881.2018.19.030 (in Chinese)

[24] Li X, Lu D P, Wang S L, Zhang M N, Lei X H, Lv X L. Design and spray characteristics simulation of an agricultural gas-liquid two-phase nozzle. Jiangsu Journal of Agricultural Sciences, 2019, 35(03): 722-728. doi: 10.3969/j.issn.1000-4440.2019.03.031 (in Chinese)

[25] Liu X Z, Gao G J. Research on atomization characteristics based on Hartmann whistle ultrasonic nozzle. Journal of Vacuum Science and Technology, 2016, 36(03): 268-272. doi: 10.13922/j.cnki.cjovst.2016.03.04 (in Chinese)

[26] Huang H, Yao X, Wang M Q, Wu X Q. Atomization performance test of ultrasonic atomization system. Piezoelectric \& Acoustooptic, 2004(01): 62-64. doi: 10.3969/j.issn.1004-2474.2004.01.019 . (in Chinese)

[27] Yang R F, Hong X Y. Ultrasonic cavitation bubble dynamics simulation of fluid governing equations. Applied Acoustics, 2018, 37(04): 455-461. doi: 10.11684/j.issn.1000-310X.2018.04.002. (in Chinese) 